\title{
Nonlinear dynamic analysis of fiber metal laminated beams subjected to moving loads in thermal environment
}

\section{Chang Tao Yi-Ming Fu Hong-Liang Dai}

\begin{abstract}
The present study is concerned with the nonlinear dynamic behaviors of fiber metal laminated (FML) beams subjected to moving loads in thermal environments. Based on von Kármán geometric nonlinear theory and Euler-Bernoulli beam hypothesis, the nonlinear equations of motion for the fiber-metal laminated beams under moving loads are derived by using Hamilton's principle. Galerkin method and Newmark method are employed to solve the dynamic responses of FML beam numerically. The dynamic responses at the midspan of the FML beam are obtained for various load velocity and temperature rise and the outcome results have been compared to the results with those obtained from linear solution. The influences of temperature, geometric nonlinearity, material parameters and velocity of the moving load on the dynamic responses of fiber-metal laminated beams are investigated. Numerical results indicate that the above-mentioned effects play a very important role on the dynamic responses of the beam.
\end{abstract}

Keywords: Nonlinear vibrations, fiber-metal laminated beam, moving loads, thermal environment

\section{Introduction}

Composite materials have gained widespread use in manufactures from space craft to marine vessels because of different possibilities. To overcome most of the disadvantages of advanced aluminum alloys and fiber reinforced composites, the idea of using the two materials, to form a hybrid structural material was born, then, initial fiber-metal laminates (FMLs) material, ARALL (Aramid Fiber Reinforced Aluminum Laminate) was introduced in 1978 [1]. Fiber-metal laminates are hybrid composite materials built up from interlacing layers of thin sheets of metal alloys and cross-ply of fiber reinforced polymeric materials [2] and find their applications in aerospace and 
satellite structures [3]. The use of FMLs has been shown to improve the impact behavior, damage resistance and thermal tolerance of high performance sandwich structures.

For the general dynamic problems and the dynamic responses of the fiber laminated composites, Noor [4] investigated the free vibration of the laminated plates systematically considering both experimental and analytical approaches. Adopting theoretical analysis and experiment, Gilat et al. [5] considered the dynamic responses of the metal matrix reinforcing laminated composite plates under the effect of the thermo- mechanical coupling, and solved the problem by using finite difference spatial domain method and Runge-Kutta method. Tanigawa et al. [6] solved the transient heat conduction and thermal stress problems of a nonhomogeneous plate with temperature-dependent material properties by using numerical method. Malekzadeh [7] analyzed the nonlinear transient heat transfer of extended surfaces by using a differential quadrature element method. Makhecha et al. [8] studied the dynamic analysis of laminated composite plates subjected to thermal/mechanical loads using an accurate theory. Aydogdu [9] analyzed the vibration of cross-ply laminated beams with general boundary conditions by Ritz method. Matsunaga [10] carried out the free vibration and stability of angle-ply laminated composite and sandwich plates under thermal loading.

Because of the wide applications of FML structures, it is important to study the dynamic response of FMLs subjected to moving loads. Many investigators [11-16] studied the dynamic problem of composite structures under the action of moving loads. Şimşek [17-19] studied dynamic responses of the functionally graded material beam subjected to moving load by using Newmark method. For the laminated composite structures, Malekzadeh [20] studied the three-dimensional dynamic analysis of laminated composite plates subjected to moving load by using differential quadrature method. Even though there are so many researchers studied the dynamic problems of the composite structures subjected to moving load, few literatures investigated the nonlinear dynamic problems of the FML beam subjected to moving loads in thermal environment. 

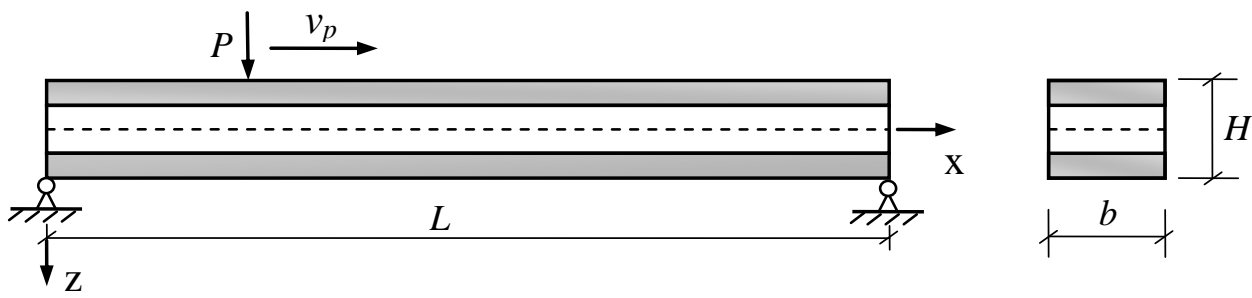

Fig. 1 Structural schematic for a fiber-metal laminated beam subjected to a moving load.

Based on Euler-Bernoulli beam theory, the displacement field can be given as

$$
\tilde{u}(x, z, t)=u(x, t)-z \frac{\partial w}{\partial x}
$$




$$
\tilde{w}(x, z, t)=w(x, t)
$$

where, $\tilde{u}, \tilde{w}$ are the displacement components of an arbitrary point in the beam along the $x, z$ coordinate axes; $u$ and $w$ are the displacement along the $x$ direction and the lateral displacement of the corresponding point in the mid-plane of the beam, respectively.

Based on the von Kármán geometric nonlinearity, the strain-displacement relations are given as

$$
\varepsilon_{x x}=\frac{\partial u}{\partial x}+\frac{1}{2}\left(\frac{\partial w}{\partial x}\right)^{2}
$$

The constitutive relation of the $k$-th layer after the coordinate transformation of the fiber-reinforced composite layer in thermal environment can be written as

$$
\sigma_{x}^{(k)}=Q_{11}^{(k)}\left(\varepsilon_{x}^{(k)}-\alpha_{x}^{(k)} \Delta T\right)
$$

where, $\Delta T$ represents the temperature rise from stress free state $\left(T=0^{\circ} \mathrm{C}\right)$, and the linear expansion coefficient $\alpha_{x}^{(k)}$, the stiffness coefficient $Q_{11}^{(k)}$ along the $x$-direction of the $k$-th layer are

$$
\begin{gathered}
\alpha_{x}^{(k)}=\alpha_{1} \cos ^{2} \theta_{k}+\alpha_{2} \sin ^{2} \theta_{k} \\
Q_{11}^{(k)}=E_{11} \cos ^{4} \theta_{k}+2\left(E_{12}+2 E_{66}\right) \cos ^{2} \theta_{k} \sin ^{2} \theta_{k}+E_{22} \sin ^{4} \theta_{k}
\end{gathered}
$$

respectively, where, $\alpha_{1}$ and $\alpha_{2}$ are the linear expansion coefficients along the fiber direction and perpendicular to the fiber direction, respectively. $\theta_{k}$ is the angle of the $k$-th lamina orientation and the elastic modulus $E_{i j}$ are given by follows:

$$
\begin{array}{ll}
E_{11}=\frac{E_{1}}{1-v_{12} v_{21}}, & E_{22}=\frac{E_{2}}{1-v_{12} v_{21}} \\
E_{12}=\frac{v_{12} E_{2}}{1-v_{12} v_{21}}, & E_{66}=G_{12}, \quad v_{21} E_{1}=v_{12} E_{2}
\end{array}
$$

where, $E_{1}$ and $E_{2}$ represent the elastic modulus along the fiber direction and perpendicular to the fiber direction, respectively, and the longitudinal and transverse Poisson's ratios are $v_{12}, v_{21}$, respectively, and $G_{12}$ is the transverse shear modulus.

For the isotropic metal layer, one gets

$$
Q_{11}^{(k)}=E_{m}, \quad v_{12}=v_{21}=v_{m}, G_{12}=\frac{E_{m}}{2\left(1+v_{m}\right)}
$$

where, $E_{m}$ and $v_{m}$ represent the elastic modulus and Poisson's ratio of the metal layer. 
From Eqs. (1), (2), (3) and (4), the kinetic energy $K$ and potential energy $U$ of FML beam can be expressed as

$$
\begin{aligned}
& K=\frac{b}{2} \int_{0}^{L} \int_{-\frac{H}{2}}^{\frac{H}{2}} \rho^{(k)}\left[\left(\frac{\partial u}{\partial t}-z \frac{\partial^{2} w}{\partial x \partial t}\right)^{2}+\left(\frac{\partial w}{\partial t}\right)^{2}\right] \mathrm{d} z \mathrm{~d} x \\
& U=\frac{b}{2} \int_{0}^{L} \int_{-\frac{H}{2}}^{\frac{H}{2}}\left[\begin{array}{l}
Q_{11}^{(k)}\left(\frac{\partial u}{\partial x}+\frac{1}{2}\left(\frac{\partial w}{\partial x}\right)^{2}-z \frac{\partial^{2} w}{\partial x^{2}}\right)^{2} \\
-2 Q_{11}^{(k)}\left(\frac{\partial u}{\partial x}+\frac{1}{2}\left(\frac{\partial w}{\partial x}\right)^{2}-z \frac{\partial^{2} w}{\partial x^{2}}\right) \alpha^{(k)} \Delta T
\end{array}\right] \mathrm{d} z \mathrm{~d} x
\end{aligned}
$$

The work of the moving load can be written as

$$
W=\int_{0}^{L} P \delta\left(x-v_{p} t\right) w(x, t) \mathrm{d} x
$$

where $\delta(\cdot)$ is the Dirac delta function. $P$ is the concentrated force of constant magnitude and $v_{p}$ is the constant velocity of the load motion. The following relation hold for the Dirac function $(c, d, \xi$ denote constants and $f(x)$ is a continuous function in the interval $<c, d>$ ):

$$
\int_{c}^{d} \delta(x-\xi) f(x) \mathrm{d} x=\left\{\begin{array}{ccc}
0 & \text { for } & \xi<c<d \\
f(\xi) & \text { for } & c<\xi<d \\
0 & \text { for } & c<d<\xi
\end{array}\right.
$$

The generalized Hamilton's principle is

$$
\delta \int_{t_{1}}^{t_{2}}(K-U) \mathrm{d} t+\int_{t_{1}}^{t_{2}} \delta W \mathrm{~d} t=0
$$

Then the nonlinear dynamic equations of motion for FML beam can be formulated and written as

$$
\begin{gathered}
I \frac{\partial^{2} u}{\partial t^{2}}=\frac{\partial N}{\partial x} \\
I \frac{\partial^{2} w}{\partial t^{2}}=\frac{\partial^{2} M}{\partial x^{2}}+\frac{\partial}{\partial x}\left(N \cdot \frac{\partial w}{\partial x}\right)+P \delta\left(x-v_{p} t\right)
\end{gathered}
$$

where $I=b \int_{-\frac{H}{2}}^{\frac{H}{2}} \rho^{(k)} \mathrm{d} z$, and $\rho^{(k)}$ is the density of the $k$-th layer.

Introducing those terms:

$$
\left\{A_{11}, B_{11}, D_{11}\right\}=b \int_{-\frac{H}{2}}^{\frac{H}{2}} Q_{11}^{(k)}\left\{1, z, z^{2}\right\} \mathrm{d} z
$$




$$
\left(\begin{array}{c}
N \\
M
\end{array}\right)=\left(\begin{array}{ll}
A_{11} & B_{11} \\
B_{11} & D_{11}
\end{array}\right)\left(\begin{array}{c}
u_{, x}+\frac{1}{2} w_{, x}^{2} \\
w_{, x x}
\end{array}\right)-\left(\begin{array}{c}
N^{T} \\
M^{T}
\end{array}\right)
$$

where, $N^{T}, M^{T}$ are the thermal force and moment, respectively, and defined as

$$
\left(N^{T}, M^{T}\right)=\int_{-\frac{H}{2}}^{\frac{H}{2}} Q_{11}^{(k)} \alpha^{(k)} \Delta T \cdot(1, z) \mathrm{d} z
$$

one can get $M^{T}=0$ after considering the symmetry.

Ignoring the in-plane inertia terms, Eq. (14) can be rewritten as

$$
\frac{\partial N}{\partial x}=0
$$

Combining Eq. (17) and Eq. (19), one can get

$$
N=N_{0}=A_{11}\left(\frac{\partial u}{\partial x}+\frac{1}{2}\left(\frac{\partial w}{\partial x}\right)^{2}\right)-N^{T}
$$

Considering the immovable ends and integrating Eq. (20) along the $x$ axis, one can get

$$
N=N_{0}=\frac{A_{11}}{2 L} \int_{0}^{L} \frac{\partial^{2} w}{\partial x^{2}} \mathrm{~d} x-N^{T}
$$

Then the nonlinear dynamic governing equations (14) and (15) can be formulated and rewritten as

$$
I \frac{\partial^{2} w}{\partial t^{2}}+D_{11} \frac{\partial^{4} w}{\partial x^{4}}+N^{T} \frac{\partial^{2} w}{\partial x^{2}}-\frac{A_{11}}{2 L} \int_{0}^{L}\left(\frac{\partial w}{\partial x}\right)^{2} \mathrm{~d} x \cdot \frac{\partial^{2} w}{\partial x^{2}}=P \delta\left(x-v_{p} t\right)
$$

The boundary conditions are

$$
\begin{aligned}
& x=0: w=0, M=0 \\
& x=L: w=0, M=0
\end{aligned}
$$

The initial condition is

$$
w(z, 0)=0
$$

\section{Solution method}

Assuming that the deflection of the FML beam can be approximately expressed as 


$$
w(x, t)=q(t) \Phi(x)
$$

where $\Phi(x)$ are the test functions which satisfied with the boundary conditions (23) and can be expressed as $\Phi(x)=\sin \left(\frac{\pi}{L} x\right)$.

Applying the Galerkin's procedure to the nonlinear dynamic equation (22), one can get

$$
M \ddot{q}+K^{\text {linear }} q+K^{\text {non }} q^{3}=F
$$

where

$$
\begin{aligned}
& M=I \int_{0}^{L} \Phi^{2} \mathrm{~d} x \\
& K^{\text {linear }}=D_{11} \int_{0}^{L} \Phi \Phi^{\prime \prime \prime \prime} \mathrm{d} x+N^{T} \int_{0}^{L} \Phi \Phi^{\prime \prime} \mathrm{d} x \\
& K^{n o n}=-\frac{A_{11}}{2 L} \int_{0}^{L} \Phi \int_{0}^{L} \Phi^{\prime \prime 2} \mathrm{~d} x \Phi^{\prime \prime} \mathrm{d} x \\
& F=\left\{\begin{array}{cc}
\int_{0}^{L} \Phi P \delta(x-v t) \mathrm{d} x=P \Phi(v t) & \text { for } 0 \leq t \leq L / v \\
0 & \text { for } t>L / v
\end{array}\right.
\end{aligned}
$$

The time-domains are discretized by Newmark method, the inertia terms can be expressed as

$$
\begin{aligned}
& (\ddot{q})_{J}=\frac{4}{(\Delta \tau)^{2}}\left[(q)_{J}-(q)_{J-1}\right]-\frac{4}{\Delta \tau}(\dot{q})_{J-1}-(\ddot{q})_{J-1} \\
& (\dot{q})_{J}=(\dot{q})_{J-1}+\frac{1}{2}\left[(\ddot{q})_{J-1}+(\ddot{q})_{J}\right] \Delta \tau
\end{aligned}
$$

The whole equations are solved iteratively. In each iterative step $J$, the nonlinear terms in the nonlinear dynamic equations (26) are linearized:

$$
(x \cdot y)_{J}=(x)_{J} \cdot(y)_{J_{P}}
$$

where $(y)_{J_{P}}$ is the average value of those obtained in the previous two iterations. For the initial iterative step, it can be determined by using the quadratic extrapolation, that is,

$$
(y)_{J_{P}}=A(y)_{J-1}+B(y)_{J-2}+C(y)_{J-3}
$$

For the different iterative steps, the coefficients A, B and C are 


$$
\begin{array}{llll}
J=1: & A=1, & B=0, & C=0 \\
J=2: & A=2, & B=-1, & C=0 \\
J \geq 3: & A=3, & B=-3, & C=1
\end{array}
$$

The iteration process goes until the difference between the results of two adjacent iteration steps is lower than $0.001 \%$, which means that the $J$ th step is convergent, then the $(J+1)$ th step can start.

\section{Numerical investigations and results}

To validate the results of the present model, an isotropic beam subjected to a moving load is investigated and compared with the analytical result. The linear analytical result can be written as

$$
\begin{gathered}
w(x, t)=\sum_{i=1}^{\Delta} \frac{2 P}{\rho b H L} \frac{1}{\left(i \pi v_{p} / L\right)^{2}-\omega_{i}^{2}}\left(\omega_{i} \sin \frac{i \pi v_{p}}{L} t-\frac{i \pi v_{p}}{L} \sin \omega_{i} t\right) \sin \frac{i \pi}{L} x \\
\left(0 \leq t \leq \frac{L}{v_{p}}\right)
\end{gathered}
$$

where

$$
\omega_{i}=\left(\frac{i \pi}{L}\right)^{2} \sqrt{\frac{E_{m} H^{2}}{12 \rho_{m}}} \quad i=1,2, \ldots, \Delta
$$

The material is Aluminum 2024-T3 and the parameters are shown in Table 1. The geometric parameters are $L=10 \mathrm{~m}, b=0.4 \mathrm{~m}, H=0.5 \mathrm{~m}$, and the velocity of the moving load is $v_{p}=40 \mathrm{~m} / \mathrm{s}$ and take $\Delta=40$. The comparisons show that the present dynamic deflections are in good agreement with the analytical result which indicate the validity of the present method. 


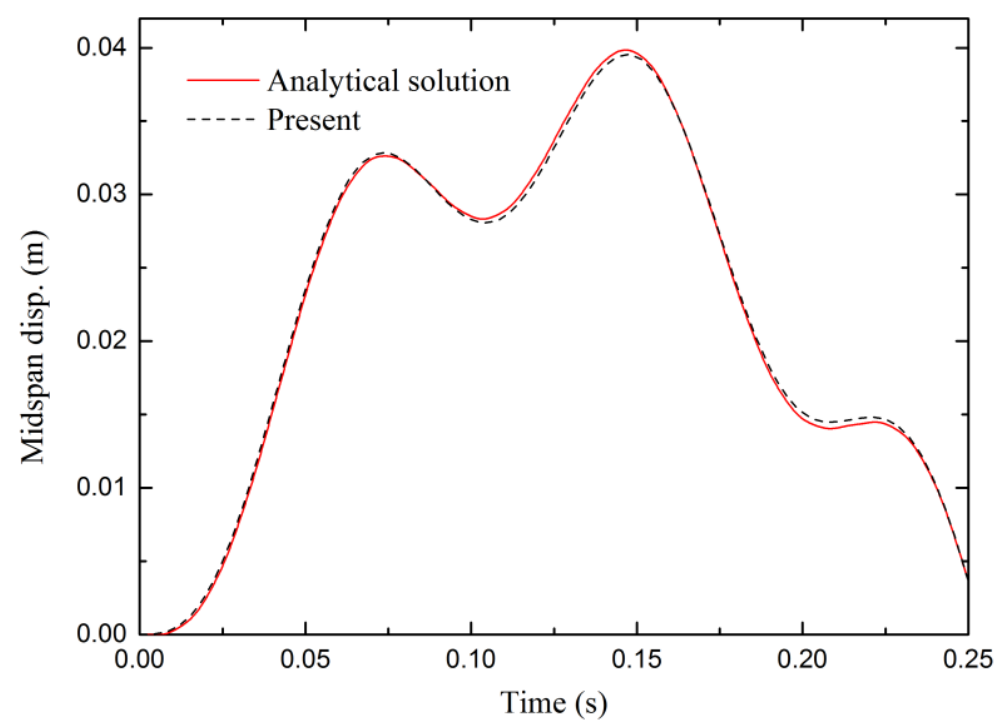

Fig. 2 Comparison of the midspan deflection of the isotropic beam by the present method to the result obtained by the analytical method.

In the following numerical calculations, a metal-fiber laminated beam with five layers is investigated: whose upper and lower layers are metal layers and fiber reinforced layer is three-layer $\left[90^{\circ} / 0^{\circ} / 90^{\circ}\right]$ symmetric cross-ply. The metal in the beam is aluminum 2024-T3 and the three fiber layers are glass-polymer composite, whose material properties are listed in Tables 1-2.

Table 1 Material properties of aluminum 2024-T3 layer.

\begin{tabular}{ccccc}
\hline Materials & $E_{m}(\mathrm{GPa})$ & $v_{m}$ & $\rho_{m}\left(\mathrm{~kg} / \mathrm{m}^{3}\right)$ & $\alpha_{m}(/ \mathrm{K})$ \\
\hline Aluminum 2024-T3 & 72.4 & 0.33 & $2.77 \times 10^{3}$ & $22.4 \times 10^{-6}$ \\
\hline
\end{tabular}

Table 2 Material properties of glass-polymer composite.

\begin{tabular}{cccccccc}
\hline Materials & $E_{1}(\mathrm{GPa})$ & $E_{2}(\mathrm{GPa})$ & $G_{12}(\mathrm{GPa})$ & $v_{12}$ & $\rho_{f}\left(\mathrm{~kg} / \mathrm{m}^{3}\right)$ & $\alpha_{1}(/ \mathrm{K})$ & $\alpha_{2}(/ \mathrm{K})$ \\
\hline $\begin{array}{c}\text { Glass-Polymer } \\
\text { Composite }\end{array}$ & 50 & 15.2 & 4.7 & 0.254 & $\begin{array}{l}2.0 \times \\
10^{3}\end{array}$ & $\begin{array}{r}6.34 \\
\times 10^{-6}\end{array}$ & $\times 10^{-6}$ \\
\hline
\end{tabular}

Figure 3 shows the linear and nonlinear time responses of the deflections at 

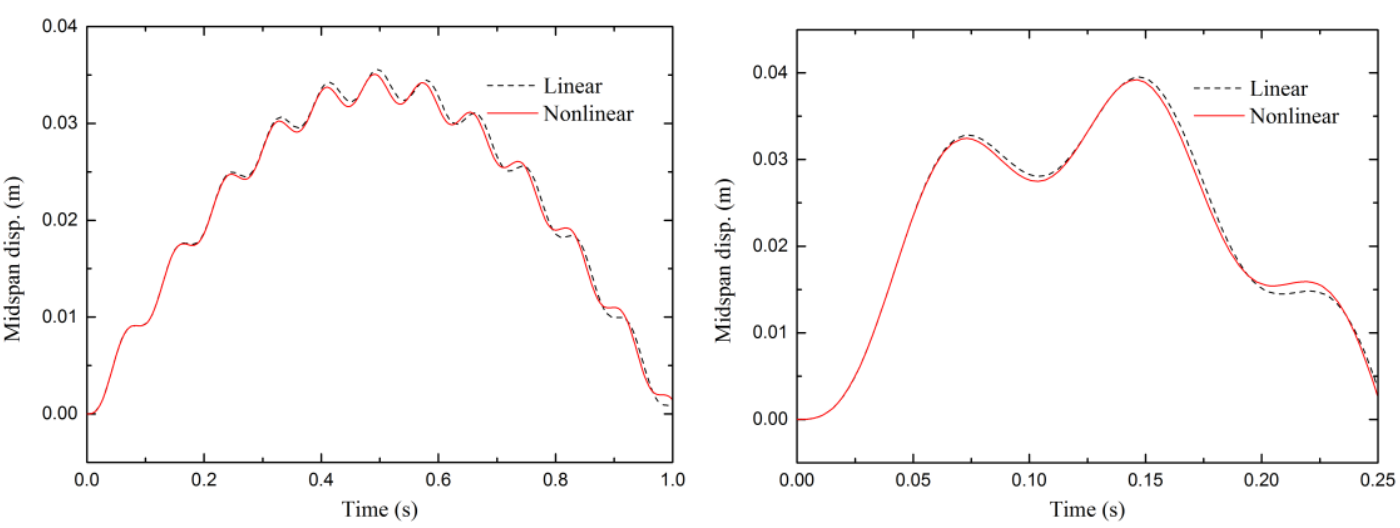

(a) $v_{p}=10 \mathrm{~m} / \mathrm{s}$

(b) $v_{p}=40 \mathrm{~m} / \mathrm{s}$
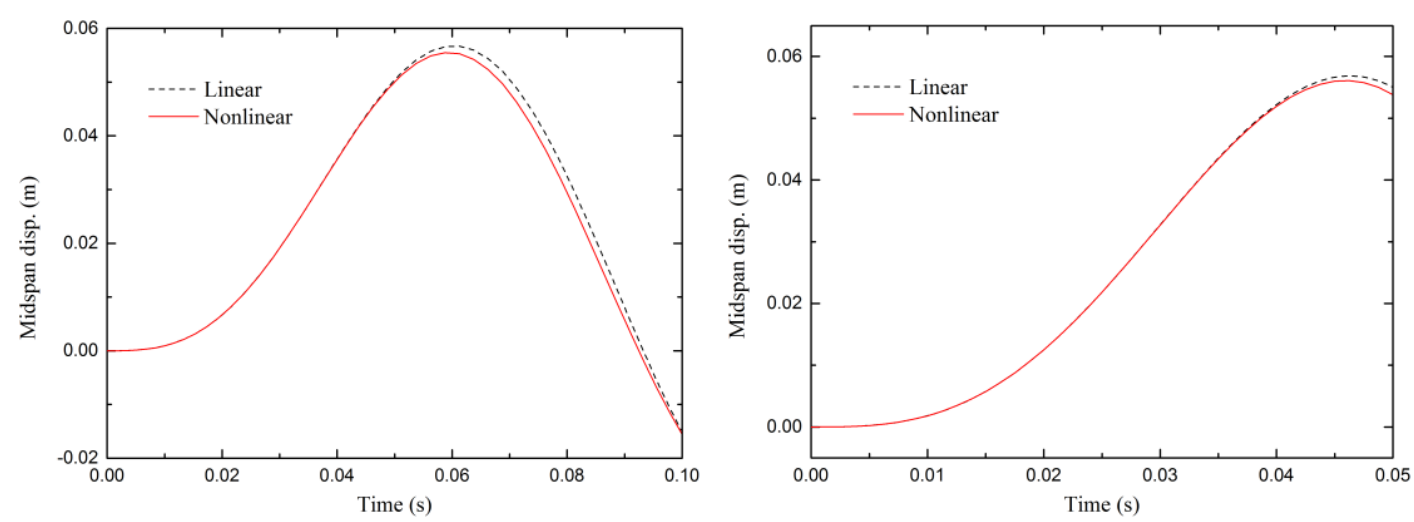

(c) $v_{p}=100 \mathrm{~m} / \mathrm{s}$

(d) $v_{p}=200 \mathrm{~m} / \mathrm{s}$

Fig. 3 Linear and nonlinear dynamic deflections at midspan of the FML beam subjected to concentrated moving loads with various velocities $(\Delta T=0 \mathrm{~K})$. 


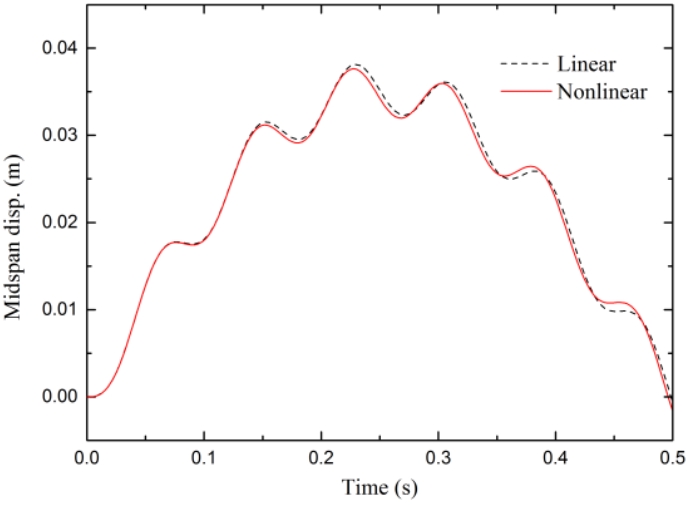

(a) $\Delta T=0 \mathrm{~K}$

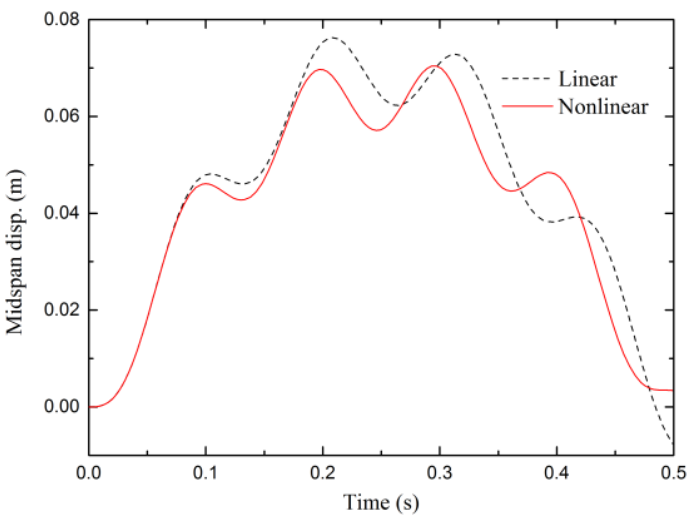

(c) $\Delta T=60 \mathrm{~K}$

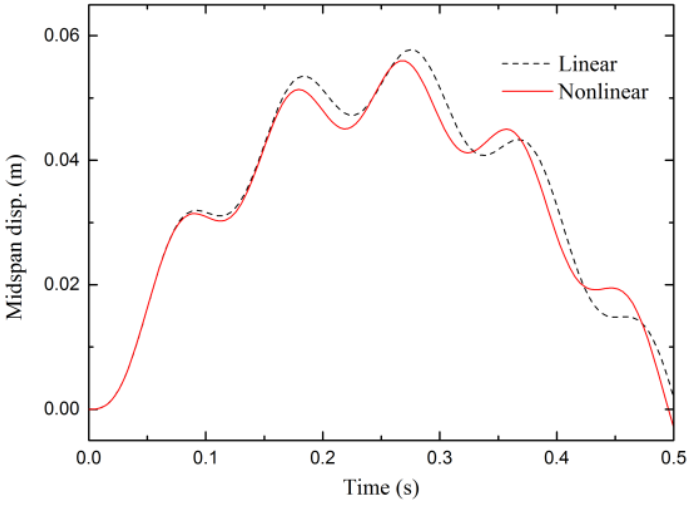

(b) $\Delta T=40 \mathrm{~K}$

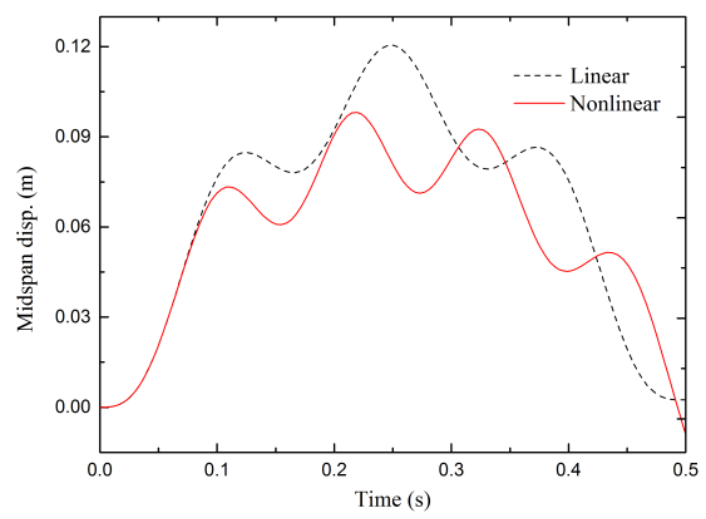

(d) $\Delta T=80 \mathrm{~K}$

Fig. 4 Linear and nonlinear dynamic deflections at midspan of the FML beam subjected to concentrated moving loads for various temperature rises $\left(v_{p}=20 \mathrm{~m} / \mathrm{s}\right)$.

The linear and nonlinear maximum normalized dynamic deflections of the midpoint of the FML beam for various values of velocity are plotted in Fig. 5. The dynamic 
deflections of the considered beam are normalized by the static deflection $w_{0}=$ $P L^{3} / 48 D_{11}$. It can be observed that the linear and nonlinear normalized dynamic deflections at midspan generally rise until a certain value of the moving load velocity, and after this value, increase in the velocity leads to a decrease in the normalized dynamic deflections. This value of the moving load velocity is called critical velocity [19]. The dynamic deflection is less than the static deflection when the velocity increases to a very high value. One can also found that the linear maximum dynamic deflections are bigger than the nonlinear maximum dynamic deflections which indicate that the internal axial displacement in the beam contributes to the lateral stiffness resulting from the geometric nonlinearity.

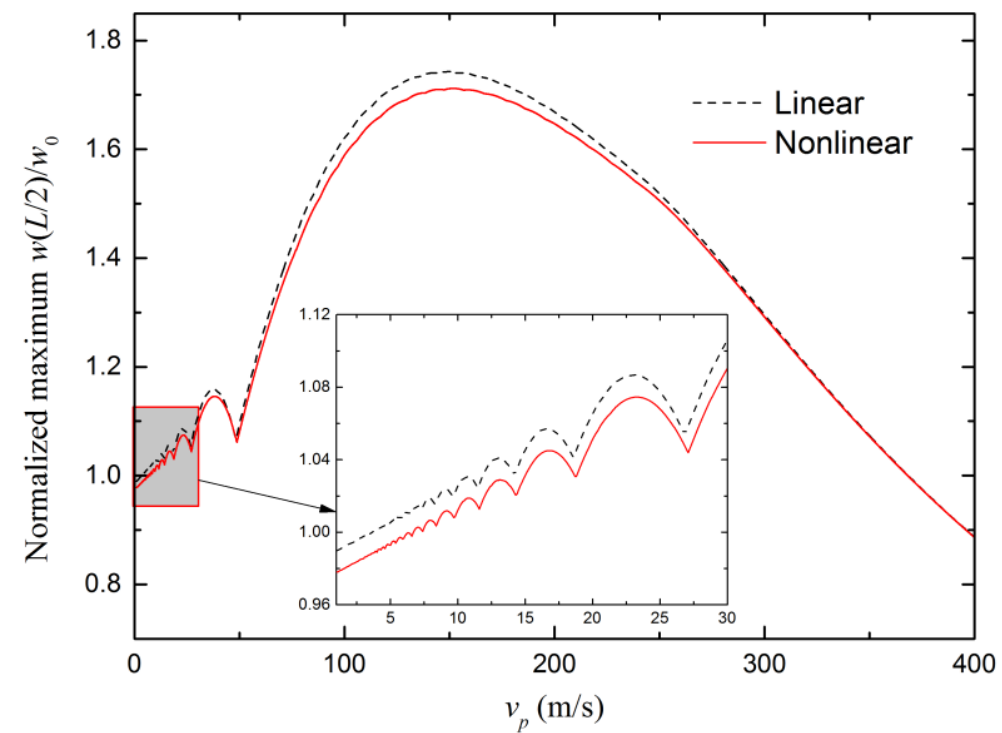

Fig. 5 Linear and nonlinear maximum normalized deflections at midspan of the FML beam with the moving load velocity.

The nonlinear maximum normalized dynamic deflections at midspan of the FML beam with the moving load velocity for various temperature reses $0 \mathrm{~K}, 20 \mathrm{~K}, 40 \mathrm{~K}$ and $60 \mathrm{~K}$ are shown in Fig. 6. It can be found that the dynamic deflection is smaller when the temperature rise is bigger which indicate that the temperature rise will decrease the stiffness of the beam. From these figures one can also see that the critical velocity decreases with the increase in the temperature. 


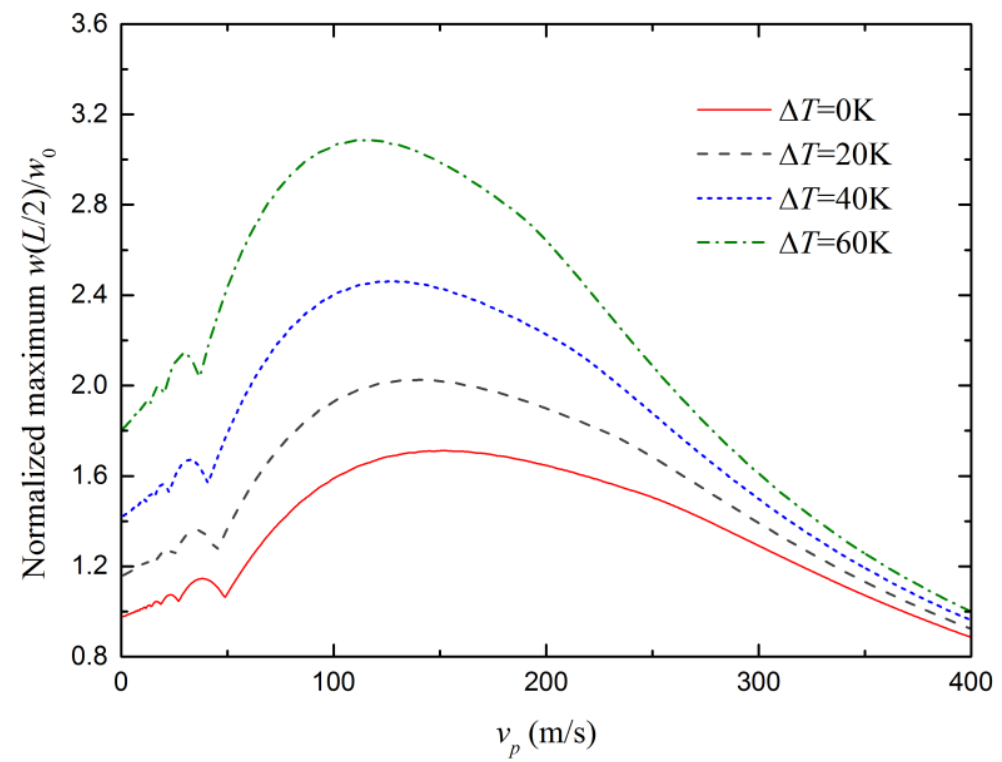

Fig. 6 Nonlinear maximum normalized dynamic deflections at midspan of the FML beam with the moving load velocity for various temperature rises.

Figure 7 and 8 show the effects of the magnitude of the moving load and the temperature rise on the linear and nonlinear maximum dynamic deflections at midspan of the FML beam. It is obvious that the nonlinear dynamic deflections are smaller than the linear dynamic deflections, and whether the magnitude of the moving load or the temperature rise increases, the difference between the linear and nonlinear deflections increases. This is due to the fact that the internal axial force in the beam contributes to the lateral stiffness resulting from the geometric nonlinearity. 


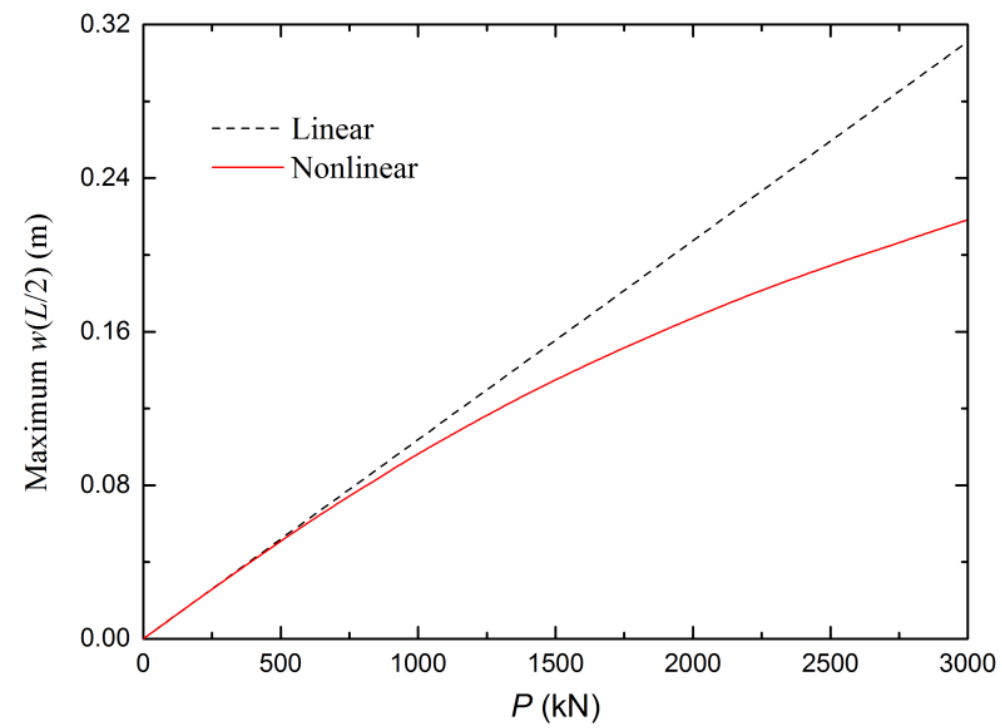

Fig. 7 Linear and nonlinear maximum dynamic deflections at midspan of the FML beam with the magnitude of the moving load.

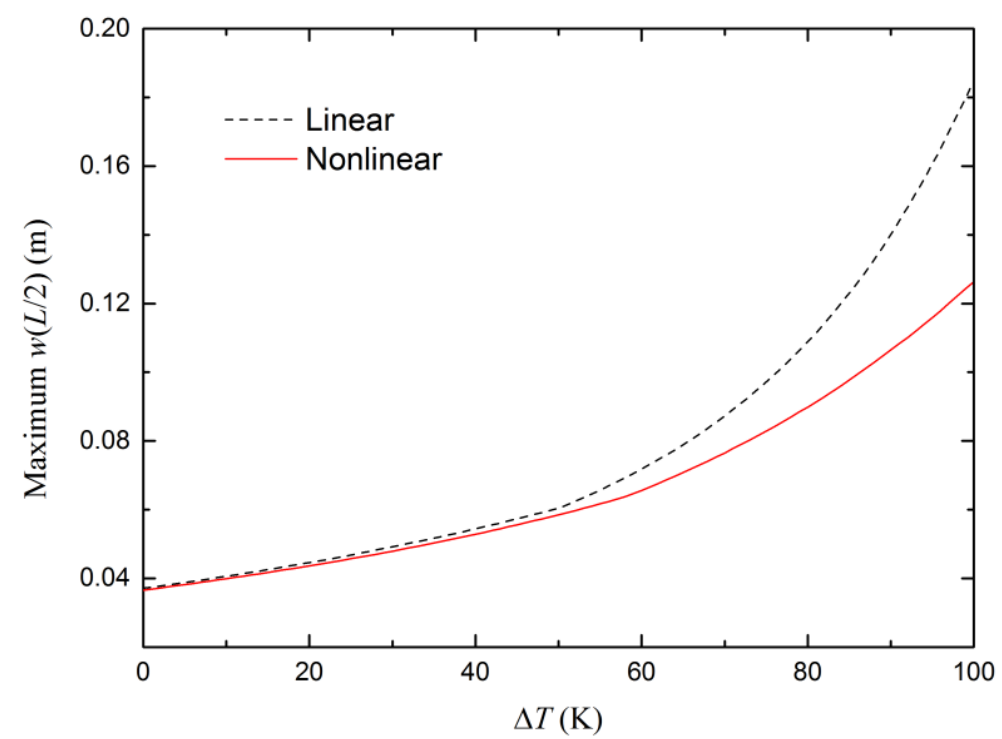

Fig. 8 Linear and nonlinear maximum dynamic deflections at midspan of the FML beam with the temperature rise.

Time responses of the dynamic deflections at midspan of FML beam subjected to concentrated moving loads for various temperature rises are shown in Fig. 9. It is clear to see that free vibration occurs when the loads leave the beam. The period of the free vibration increases when the temperature rises, which indicate that the axial stress decreases the stiffness of the beam. 


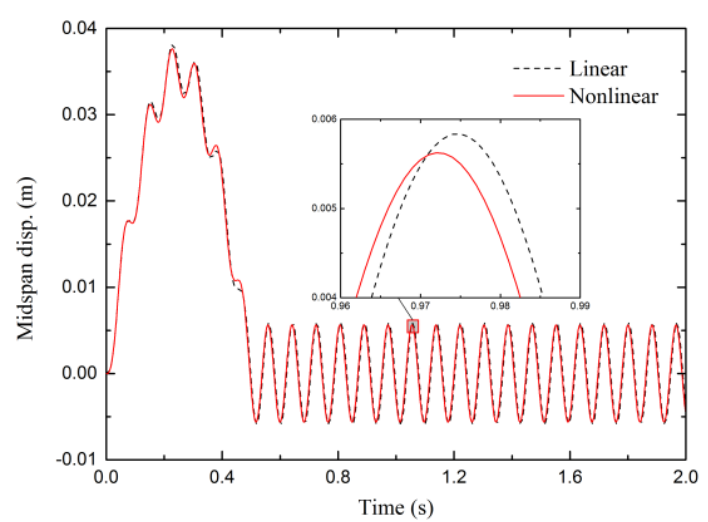

(a) $\Delta T=0 \mathrm{~K}$

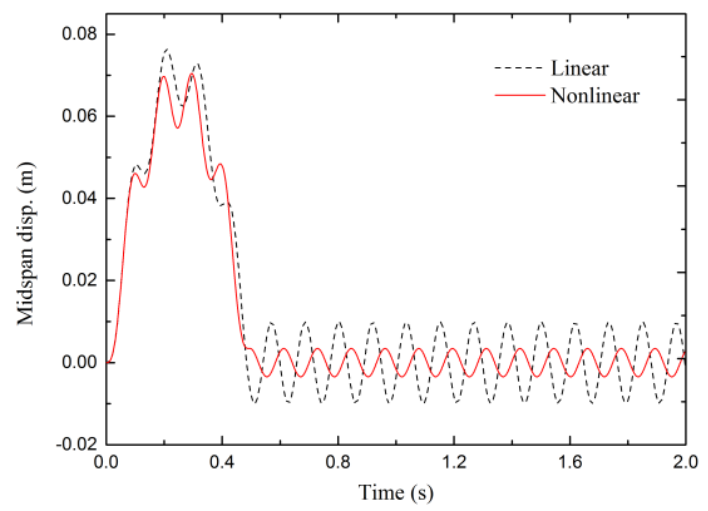

(c) $\Delta T=60 \mathrm{~K}$

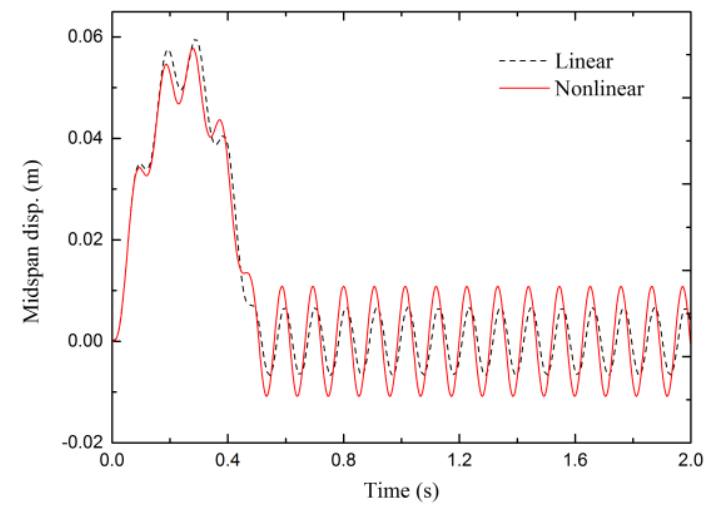

(b) $\Delta T=40 \mathrm{~K}$

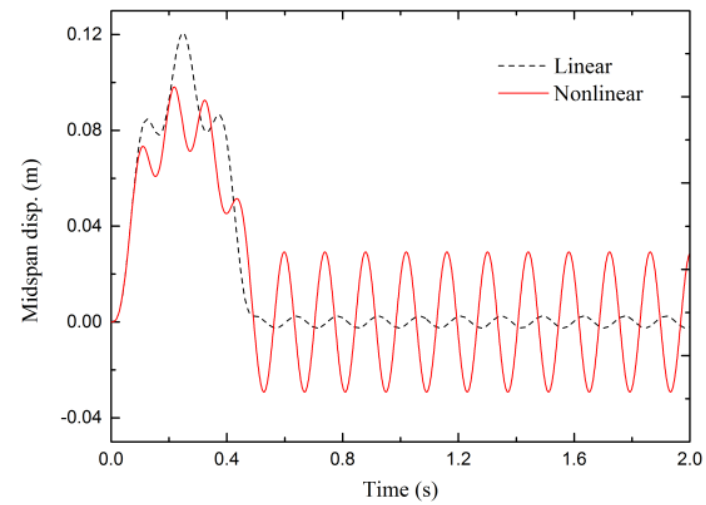

(d) $\Delta T=80 \mathrm{~K}$

Fig. 9 Time responses of the dynamic deflections at midspan of FML beam subjected to concentrated moving loads for various temperature rises $\left(v_{p}=20 \mathrm{~m} / \mathrm{s}\right)$.

\section{Conclusions}

The present study is concerned with the nonlinear dynamic behaviors of fiber metal laminated (FML) beams subjected to moving loads in thermal environments. Based on von Kármán geometric nonlinear theory and Euler-Bernoulli beam hypothesis, the nonlinear governing equations of motion for the fiber-metal laminated beams under moving loads are derived. The influences of temperature, geometric nonlinearity, material parameters and velocity of the moving load on the dynamic responses of fiber-metal laminated beams are investigated. The main conclusions are as follows: geometric linear and nonlinear results have little difference and the difference changes little with the increase of the velocity of the moving loads; the maximum deflection at 


\section{Acknowledgement}

This study is supported by the National Science Foundation of China under Grant No. 11272117.

\section{References}

[1] L. Vogelesang, A. Vlot, Development of fibre metal laminates for advanced aerospace structures, Journal of Materials Processing Technology, 103 (2000) 1-5.

[2] P. Cortes, W. Cantwell, The prediction of tensile failure in titanium-based thermoplastic fibre-metal laminates, Composites science and technology, 66 (2006) 2306-2316.

[3] V. Pradeep, N. Ganesan, K. Bhaskar, Vibration and thermal buckling of composite sandwich beams with viscoelastic core, Composite Structures, 81 (2007) 60-69.

[4] A.K. Noor, Free vibrations of multilayered composite plates, AIAA journal, 11 (1973) 1038-1039.

[5] R. Gilat, J. Aboudi, Thermomechanical coupling effects on the dynamic inelastic response and buckling of metal matrix composite infinitely wide plates, Composite Structures, 35 (1996) 49-63.

[6] Y. Tanigawa, T. Akai, R. Kawamura, N. Oka, Transient heat conduction and thermal stress problems of a nonhomogeneous plate with temperature-dependent material properties, Journal of Thermal Stresses, 19 (1996) 77-102.

[7] P. Malekzadeh, H. Rahideh, G. Karami, A differential quadrature element method for nonlinear transient heat transfer analysis of extended surfaces, Numerical Heat Transfer, Part A: Applications, 49 (2006) 511-523.

[8] D. Makhecha, M. Ganapathi, B. Patel, Dynamic analysis of laminated composite plates subjected to thermal/mechanical loads using an accurate theory, Composite 
Structures, 51 (2001) 221-236.

[9] M. Aydogdu, Vibration analysis of cross-ply laminated beams with general boundary conditions by Ritz method, International Journal of Mechanical Sciences, 47 (2005) 1740-1755.

[10] H. Matsunaga, Free vibration and stability of angle-ply laminated composite and sandwich plates under thermal loading, Composite Structures, 77 (2007) 249-262.

[11] M.R. Taheri, E.C. Ting, Dynamic response of plate to moving loads: structural impedance method, Computers \& structures, 33 (1989) 1379-1393.

[12] M.R. Taheri, E.C. Ting, Dynamic response of plates to moving loads: finite element method, Computers \& structures, 34 (1990) 509-521.

[13] M.-H. Huang, D.P. Thambiratnam, Dynamic response of plates on elastic foundation to moving loads, Journal of Engineering Mechanics, 128 (2002) 1016-1022.

[14] X. Zhu, S. Law, Dynamic behavior of orthotropic rectangular plates under moving loads, Journal of Engineering Mechanics, 129 (2003) 79-87.

[15] J.-J. Wu, Dynamic analysis of a rectangular plate under a moving line load using scale beams and scaling laws, Computers \& structures, 83 (2005) 1646-1658.

[16] S. Law, J. Bu, X. Zhu, S. Chan, Moving load identification on a simply supported orthotropic plate, International Journal of Mechanical Sciences, 49 (2007) 1262-1275.

[17] M. Şimşek, T. Kocatürk, Ş. Akbaş, Dynamic behavior of an axially functionally graded beam under action of a moving harmonic load, Composite Structures, 94 (2012) 2358-2364.

[18] M. Şimşek, Vibration analysis of a functionally graded beam under a moving mass by using different beam theories, Composite Structures, 92 (2010) 904-917.

[19] M. Şimşek, Non-linear vibration analysis of a functionally graded Timoshenko beam under action of a moving harmonic load, Composite Structures, 92 (2010) 2532-2546.

[20] P. Malekzadeh, A. Fiouz, H. Razi, Three-dimensional dynamic analysis of laminated composite plates subjected to moving load, Composite Structures, 90 (2009) $105-114$. 